\title{
PRODUCTION OF MICROEMULSION BY MEMBRANE EMULSIFICATION: COMPARISON OF EMPTY CERAMIC TUBE MEMBRANE AND MEMBRANE EQUIPPED WITH STATIC TURBULENCE PROMOTERS
}

\author{
K. Albert*, A. Koris, I. Gáspár, G. Rácz and Gy. Vatai \\ Department of Food Engineering, Faculty of Food Science, Corvinus University of Budapest \\ 1118 Budapest, Ménesi út. 44. Hungary
}

(Received: 15 April 2014; accepted: 30 June 2014)

\begin{abstract}
Membrane emulsification (ME) is a relatively new technique for the highly controlled production of particulates, which helps to obtain a narrower distribution compared to other emulsification techniques such as homogenizers or ultrasound. Benefits of membrane emulsification for food applications include the low shear properties and the uniform size distribution. In this process, the dispersed phase (oil) is pressed through the pores of a microporous membrane directly into the continuous phase (water) flowing tangentially to the membrane surface. The purpose of the emulsification experimentations was to find and model operating conditions of the operation. In laboratory experiments from conventional, commercial grade sunflower oil (dispersed phase) and from distilled water (solid phase), emulsions were prepared. The ceramic tube membrane with nominal pore size of $1.4 \mu \mathrm{m}$ was used in the experiments $\left(\mathrm{ZrO}_{2}\right)$. In order to increase the shear-stress near the membrane wall (influence the characteristics of the flow regime of the continuous phase), a kind of self-fabricated helical-shaped-ribbon reducer was installed inside the tube membrane.
\end{abstract}

Keywords: membrane emulsification, static turbulence promoter, modelling, oil-in-water emulsion

This work focuses on the highly controlled production of droplets. In the course of membrane emulsification, a liquid phase is pressed through the membrane pores to form droplets at the permeate side of a membrane. Emulsified droplets are formed and detached at the end of the pores with a drop-by-drop mechanism (DE LuCA et al., 2008). In order to ensure a regular droplet detachment from the pore outlets, shear stress is generated at the membrane/ continuous phase interface by recirculating the continuous phase using a pump or by agitation in a stirring vessel (VLADISAVLJEVIC \& WiLliAMS, 2005).

One of the innovative methods for generating shear at the surface of a membrane for the purpose of $\mathrm{ME}$ is by pulsing the flow over the membrane surface formed from a tube (HoLDICH et al., 2010, 2013). In literature it is reported that helical baffles are likely to perform better compared to rod inserts, implying that the helical vortices improve the mixing between the boundary layer on the surface of the membrane and the bulk fluid to a greater degree than by simply generating turbulent flow using cylindrical rod inserted (AHMAD et al., 2005).

According to these researches KORIS and co-workers (2011) established that, with a simple mechanical method, by insertion of static turbulence promoters, it was possible to increase shear stress at the membrane surface, while maintaining a low shear in the

\footnotetext{
* To whom correspondence should be addressed. Phone: +36-1-482-6113; e-mail: krisztina.albert1986@gmail.com
} 
recirculation loop. The present paper deals with a simple, static method, which is costeffective and is able to increase the productivity of cross-flow membrane emulsification with satisfying quality.

\section{Materials and methods}

\subsection{Materials and membrane}

In laboratory experiments from conventional, commercial grade sunflower oil (dispersed phase) and from distilled water solution (continuous phase), emulsions were prepared. The $1.5 \mathrm{w} / \mathrm{w} \%$ continuous phase was formed from Tween 80 (Sigma-Aldrich Chemie GmbH) organic surfactant and distilled water.

The ceramic tube membrane (Pall Schumacher Corporation) with nominal pore size of $1.4 \mu \mathrm{m}$ was used in the experiments (active $\mathrm{ZrO}_{2}$ layer). The droplet size and the droplet size distribution in the emulsion samples made with this membrane were measured by Fritsch Laser Particle Sizer Analysette 22.

\subsection{Apparatus}

A cross flow membrane emulsification system was used to produce all emulsions. The experimental set-up used for the experiments is shown in Figure 1. The apparatus is very simple; it was designed from the practical application viewpoint. Two manometers, one placed before the membrane and the other placed after the membrane, were used to measure the pressure drop through the membrane. The continuous phase was recirculated on the lumen side of the membrane by a submersible pump. The oil pressure was guaranteed by compressed air with an air pump and it was injected from the outer surface of the membrane.

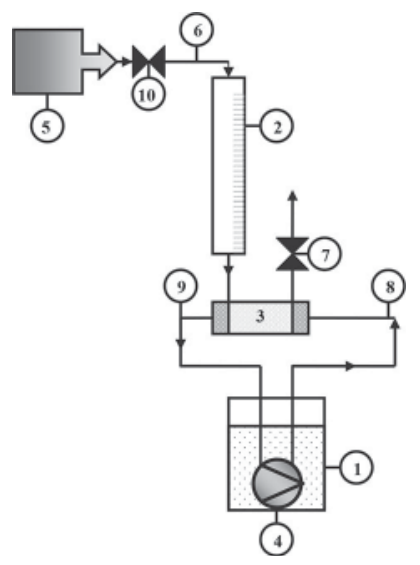

Fig. 1. Scheme of the cross-flow membrane emulsification apparatus. 1: Continuous phase tank; 2: graduated disperse phase tank; 3: membrane module + reducer; 4: submersible pump; 5: compressed air connection; 6: pressure meter; 7: valve on drain; 8: pressure meter; 9: pressure meter; 10: pressure control 


\subsection{Calculations}

The cross-flow membrane emulsification method involves transmembrane pressure to force the dispersed phase to permeate through the membrane into the continuous phase. The driving force (DF) is expressed as the ratio of transmembrane pressure (TMP) and critical pressure (CP) according to Eq. 1.

$$
\mathrm{DF}=\frac{\mathrm{TMP}}{\mathrm{CP}}
$$

The transmembrane pressure, TMP, is defined as the difference between the pressure of the dispersed phase, $P_{d}$, and the mean pressure of the continuous phase, Eq. 2:

$$
\mathrm{TMP}=P_{d}-\frac{\mathrm{P}_{\mathrm{c}, \mathrm{in}}+\mathrm{P}_{\mathrm{c}, \text { out }}}{2}
$$

where $\mathrm{P}_{c, \text { in }}$ and $\mathrm{P}_{\mathrm{c}, \text { out }}$ are the pressure of the flowing continuous phase at the inlet and at the outlet of the membrane device, respectively.

The shear stress $(\tau)$ near the lumen side of the membrane surface is calculated from Eq. 3:

$$
\tau=k \cdot \frac{\lambda \cdot \rho \cdot v^{2}}{2}[P a]
$$

The droplet uniformity was expressed as the span of droplet size distribution. A measure of the size distribution of the droplets in an emulsion given by the difference between the size at $90 \%$ and $10 \%$ of the population of droplets, divided by the size at $50 \%$ of the population:

$$
\operatorname{span}=\frac{d_{90}-d_{10}}{d_{50}} .
$$

1.3.1. Configuration with helix reducer. The constant $k$ is a geometry dependent correction coefficient for the system with non-cylindrical insert. In case of empty, cylindrical tube the value of $k$ equals 1 . To evaluate the factor $k$ for helix turbulence promoter, Eq. 5 can be used:

$$
k=\frac{\mathrm{R} \cdot \pi+\mathrm{a}+\mathrm{b}}{[\mathrm{R} \cdot \pi+\mathrm{z}(\mathrm{a}+\mathrm{b})]}
$$

where $\mathrm{a}$ and $\mathrm{b}$ are the geometric parameters of the helix turbulence promoter $(\mathrm{a}=0.00635 \mathrm{~m}$, $\mathrm{b}=0.001 \mathrm{~m})$, and $\mathrm{R}$ is the inner radius of the tube $(\mathrm{R}=0.0035 \mathrm{~m})$. The absolute length ratio of the promoter $\mathrm{z}$ is calculated by Eq. 6 : 


$$
\mathrm{z}=\frac{\mathrm{H}}{\mathrm{c}}
$$

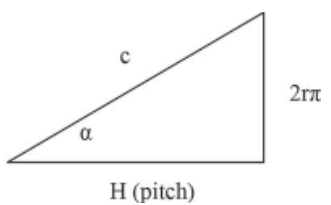

where $\mathrm{H}$ and $\mathrm{c}$ are the geometrical parameter of helix turbulence (KoRIs et al., 2011).

1.3.2. Calculation of pumping energy. The efficiency of the reducer as a turbulence promoter was also determined by evaluating the reduction of specific energy consumption (ER). One of the most important parameters from an economical point of view is the specific energy consumption, E, defined as the power dissipated per unit volume of permeate. The hydraulic dissipated power can be expressed as the product of the feed flow rate and pressure drop along the module, Eq. 7:

$$
\mathrm{P}=\mathrm{Q} \cdot \Delta \mathrm{P}
$$

where $\mathrm{P}(\mathrm{W})$ is the hydraulic dissipated power, $\mathrm{Q}\left(\mathrm{m}^{3} \mathrm{~s}^{-1}\right)$ is the feed flow rate, and $\Delta \mathrm{P}(\mathrm{Pa})$ is the pressure drop observed along the membrane.

\subsection{Membrane emulsification and experimental design}

In the emulsification experiments two parameters were changed: the pressure of disperse phase (expressed in DF-driving force) and the recirculation flow (shear stress at the wall of the membrane). The disperse phase flux was determined by volume, upon the oil consumption from the graduated feed tube by using formulas. The experiments were carried out at room temperature. The axial cross-flow velocity in the tube membranes was varied by $0.35 \mathrm{~m} \mathrm{~s}^{-1}$ and $0.7 \mathrm{~m} \mathrm{~s}^{-1}$ while the re-circulated flow-rate (RFR) was set to $50 \mathrm{l} \mathrm{h}^{-1}$ and $100 \mathrm{l} \mathrm{h}^{-1}$, respectively, on the pump. The shear-stress was observed as $0.4 \mathrm{~Pa}$ and $0.8 \mathrm{~Pa}$, accordingly. The emulsion was also prepared without any cross-flow, in which case the re-circulated flow rate and shear stress were 0 . The applied transmembrane pressure (TMP) varied between 1 and 1.8 bars.

The system and the membrane were cleaned between the experiments with ULTRASIL-11 $(\sim 1 \% \mathrm{w} / \mathrm{w})$ solution when it was necessary. In order to increase the shear-stress near the membrane wall (influence the characteristics of the flow regime of the continuous phase), two kinds of self-fabricated helical-shaped-ribbon reducers were installed inside the tube membrane (Fig. 2).

To evaluate the results, statistical methods, $3^{\mathrm{p}}$ type full factorial experimental designs, were evaluated using the software STATISTICA. In this experiment, the effect of driving force $(D F)$, shear stress $(\tau)$ (regulated by flow rate), and pore size were investigated as independent variables. The fluxes, diameter of droplets, and span were used as dependent variables. The effects of independent variables on dependent variables were observed. 


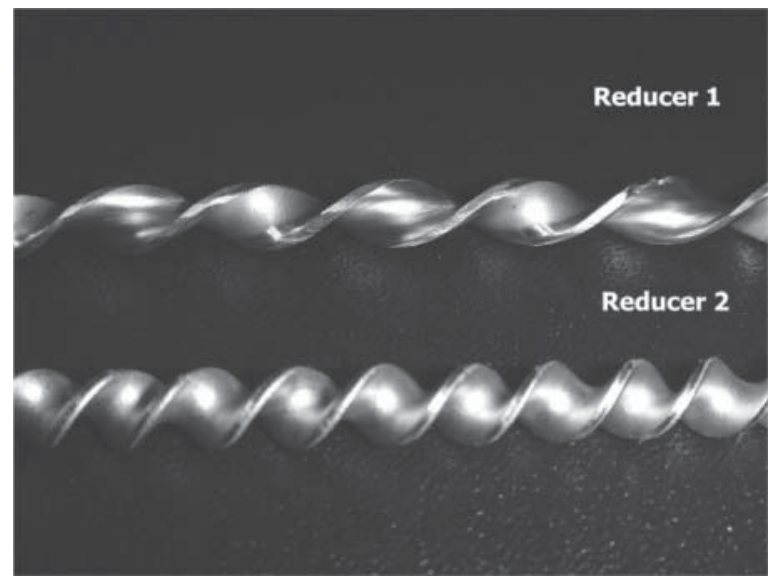

Fig. 2. Simulated figure of the helix reducers

\section{Results and discussion}

2.1. Response surface models for the effects of driving force and shear-stress on flux, droplet size, and span

The effects of driving force and shear-stress above the membrane surface can be seen in case of empty tube, using reducer 1, and in case of using reducer 2: on flux (Fig. 3), on droplet size (Fig. 4) and on span (Fig. 5). From the fitted surfaces of Fig. 3 it was found that when the turbulence promoter is inserted into the ceramic membrane channel, the fluid flow patterns are changed. It was observed that the flux was steadily increased with the increasing driving force, as transmembrane pressure is the driving force in the emulsification process. The reason for this kind of behaviour may be found in the increase in cross-flow velocity when the flow rate is increased and the accumulation of oil droplets at the membrane surface is less prominent, the dispersed phase flux increases.

A

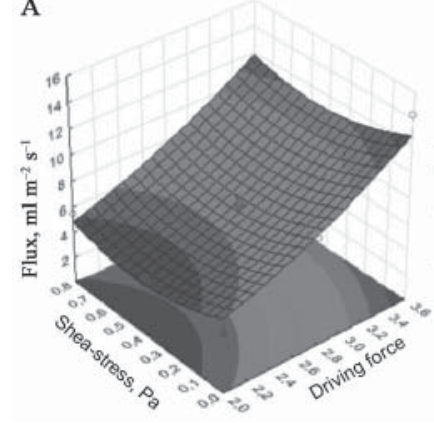

B

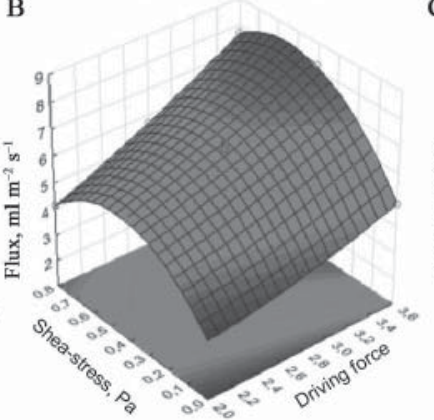

C

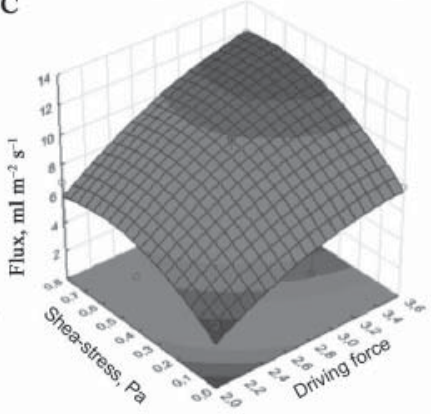

Fig. 3. Fitted 3D surfaces on the effects of driving force and shear-stress on flux: in case of empty tube (A), using reducer 1 (B), using reducer 2 (C), $1.4 \mu \mathrm{m}$ 
On the other hand, shear stress has less intensive effect on flux compared to driving force. In all three cases, the interaction of these two factors is also visible, the surfaces are slightly twisted. In case of using reducers, in Fig. 3B and 3C the same can be observed: the driving force increases the flux, while the shear-stress after the medium point $(\tau=0.4)$ causes the opposite, the flux decreases.
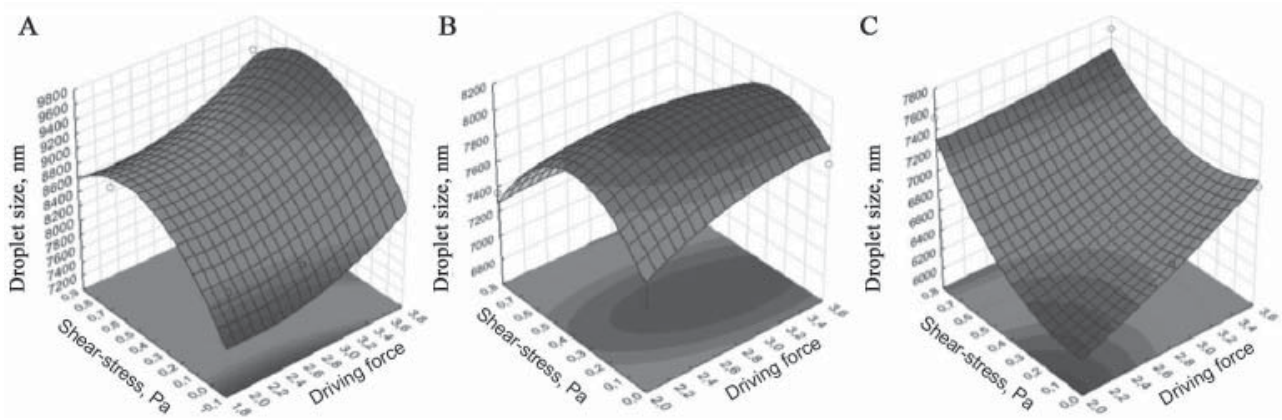

Fig. 4. Fitted $3 \mathrm{D}$ surfaces on the effects of driving force and shear-stress on droplet size: in case of empty tube (A); using reducer 1 (B); using reducer 2 (C), $1.4 \mu \mathrm{m}$

The response surface model for the effects on droplet size is shown in Figure 4, in case of empty tube (A), using reducer 1 (B), and in case of using reducer 2 (C). The figures clearly show that in case of reducers, the increase of driving force and shear-stress increases the average values of droplet size diameter. Contrary to systems using reducer, in case of an empty tube, the driving force has no significant effect on the droplet size. The effect of shearstress shows a different behaviour in the three cases. With increase of shear-stress in case of reducer 2, the increase of droplet size is apparent, while without reducer after the medium point $(\tau=0.4)$ the droplet size is almost constant, and using reducer 1 causes the opposite, the droplet size decreases. In all cases the interaction of the two factors cannot be neglected; it also has a significant effect on the size distribution of the droplets, which is visible from the twisted surfaces.
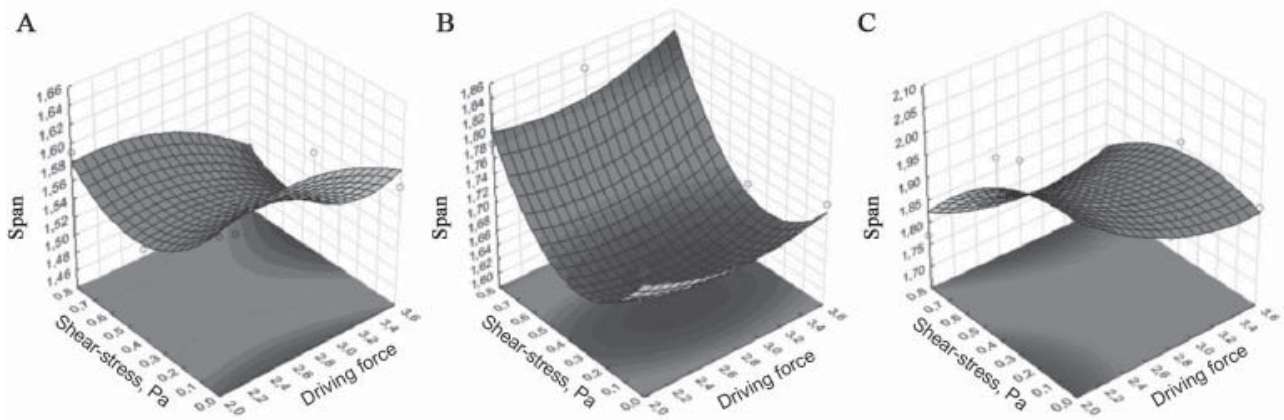

Fig. 5. Fitted 3D surfaces on the effects of driving force and shear-stress on span: in case of empty tube (A); using reducer 1 (B); using reducer 2 (C), $1.4 \mu \mathrm{m}$ 
In Fig. 5, the combination of the effects of driving force and shear-stress on span are shown in case of empty tube, using reducer 1, and in case of reducer 2. From Figure 5 it can be observed that driving force and shear stress have important effect on getting fine emulsion, their interaction is significant: the surfaces are twisted. In the first two cases, without reducer and using reducer 1 , after the medium point $(\tau=0.4)$, span is increased with increase of shearstress. On the other hand, driving force has smaller influence on the span compared to the influence of shear-stress.

From the aspect of productivity, shear stress $=0.4 \mathrm{~Pa}$ on $\mathrm{DF}=3.6$ (using reducer 2) is suggested for operation, since these conditions ensure high flux $\left(12 \mathrm{ml} \mathrm{m}^{-2} \mathrm{~s}^{-1}\right)$ with good quality emulsion (droplet size $\approx 6.8 \mu \mathrm{m}$, span $\approx 1.85$ ).

\subsection{Result of pumping power requirement calculations}

One of the most important parameters from an economical point of view is the specific energy consumption. P was calculated according to experimental data and Eq. 7. In this measurement only reducer 2 was tested and compared to non-reducer mode. The reducers increase the shear-stress near the membrane wall, so using reducer we could set the flow rate on a smaller value in order to keep the same shear stresses.

Result of pumping power requirement calculations allows concluding that, in most of the cases (except when no recirculation were applied $\left(Q_{\text {rec }}=01 \mathrm{~h}^{-1}\right)$ ), around $8 \%$ less energy is required for recirculation of the continuous phase, and the emulsion with reducer is comparable to the case when no reducer was used. In Figure 6 the anterior bars represent the energy required for preparing emulsion without using the reducer, and the rearward bars represent the energy required while the reducer was used.

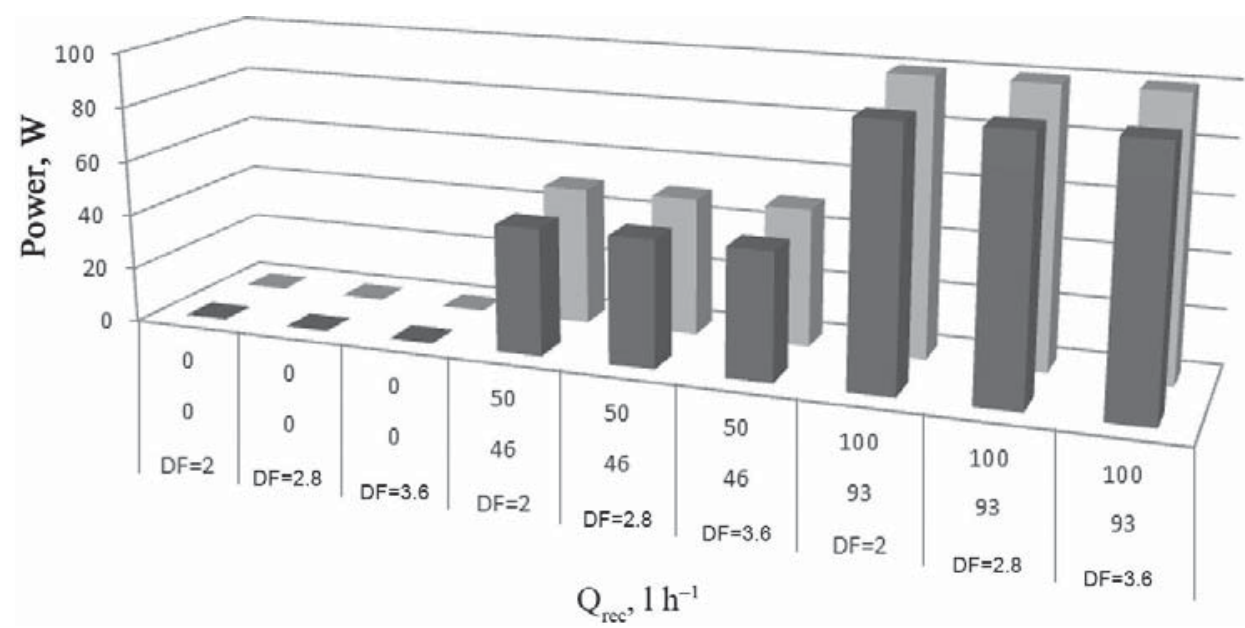

Fig. 6. Relative pumping energy consumption to recycle the continuous phase 


\section{Conclusions}

Fine quality emulsions were obtained with low span values when monodisperse $\mathrm{O} / \mathrm{W}$ emulsions were prepared using commercially available microfiltration tubular ceramic membranes. The response surface methodology proved to be an adequate modelling tool for mathematical representation of the process. For prediction of the flux, droplet size, and span, a mathematical model was set up that can well describe the dependent variables in the studied range, namely the run of the flux, the mean droplet diameter, and the effects of operating parameters. The application of helical-shaped reducer has positive effects on the emulsification process, when the turbulence promoter is inserted, fluid flow patterns change. In most of the cases, around $8 \%$ less energy was required for recirculation of the continuous phase, and the emulsion with reducer is comparable to the case when no reducer was used.

The authors would like to express their acknowledgement to the Hungarian National Scientific Foundation TÉT 10-1-2011-0072, OTKA K68596 and TÁMOP-4.2.1/B-09/1/KMR-2010-0005, and for MEMFIDA2 project (EUREKA_HU_08-1-2010-0010) for the support.

\section{References}

AhMAD, A.L., Maridas, A. \& LAU, K.K. (2005): Flux enhancement by introducing turbulence effect for microfiltration of Saccharomyces cerevisiae. Separ. Sci. Technol., 40, 1213-1225.

De Luca, G., Di Maio, F., Di Renzo, A. \& Drioli, E. (2008): Droplet detachment in cross-flow membrane emulsification: Comparison among torque and force-based models. Chem. Eng. Proc., 47, 1150-1158.

Holdich, R.G., Dragosavac, M.M., Vladisavljević, G.T. \& Kosvintsev, S.R. (2010): Membrane emulsification with oscillating and stationary membranes. Ind. Eng. Chem. Res., 49, 3810-3817.

Holdich, R.G., Dragosavac, M.M., Vladisavljević, G.T. \& Piacentini, E. (2013): Continuous membrane emulsification with pulsed (oscillatory) flow. Ind. Eng. Chem. Res., 52, 507-515.

Koris, A., Piacentini, E., Vatai, G., Bekassy-Molnar, E., Drioli, E. \& Giorno, L. (2011): Investigation on the effects of a mechanical shear-stress modification method during cross-flow membrane emulsification. J. Membrane Sci., 371, 28-36.

VladisaVlJeVIC, G.T. \& Williams, R.A. (2005): Recent developments in manufacturing emulsions and particulate products using membranes. Adv. Colloid Interfac., 113, 1-20. 